\title{
Phase II study of irinotecan combined with carboplatin in previously untreated small-cell lung cancer
}

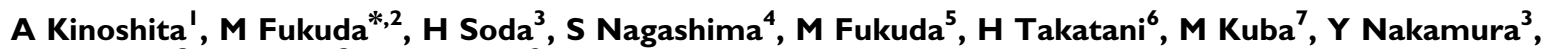 \\ J Tsurutani ${ }^{3}$, $\mathbf{S} \mathrm{Kohno}^{3}$ and $\mathrm{M} \mathrm{Oka}^{2}$ for the Nagasaki Thoracic Oncology Group (NTOG) \\ 'National Nagasaki Medical Center, Nagasaki, Japan; ${ }^{2}$ Kawasaki Medical School, Division of Respiratory Diseases, Department of Medicine, 577 \\ Matsushima, Kurashiki, Okayama 70 I-0 192, Japan; ${ }^{3}$ Second Department of Internal Medicine, Nagasaki University School of Medicine, Nagasaki, Japan; \\ ${ }^{4}$ Sasebo General Hospital, Nagasaki, Japan; 5 Japanese Red-Cross Nagasaki Atomic Bomb Hospital, Nagasaki, Japan; ${ }^{6}$ Nagasaki Municipal Hospital, \\ Nagasaki, Japan; ${ }^{7}$ National Okinawa Hospital, Okinawa, Japan
}

To determine the efficacy and toxicity of irinotecan combined with carboplatin, we conducted a phase II trial. Eligibility criteria were: chemotherapy-naïve, small-cell lung cancer (SCLC), good performance status (PS: $0-2$ ), age $\leqslant 75$ years, and adequate organ function. The patients' characteristics were: male/female $=56 / 5$; PS 0/I/2 = 19/38/4; median age (range) =68 years (5I -75 years); limited disease $(\mathrm{LD})$ /extensive disease $(E D)=27 / 34$. The patients received irinotecan $\left(50 \mathrm{mg} \mathrm{m}^{-2}\right)$ on days I, 8 , and I5, and carboplatin (AUC 5, Chatelut formula) on day I every 4 weeks. In total, $6 I$ patients were eligible and all were evaluated. In all, 3 I patients were treated with four or more courses of chemotherapy. Of the patients, 17 showed a complete response (CR), 34 showed a partial response (PR), nine had stable disease (SD), and one had progressive disease (PD). The overall response rate was $84 \%$ (95\% confidence interval (Cl), 72-91\%; LD 89\%, ED 79\%) and the CR rate was 28\% (95\% Cl, I7-41\%; LD 37\%, ED 21\%). The median time to tumour progression was 6.I (LD 6.4, ED 5.4) months. The medial survival time was I5.0 (LD 20.0, ED 9.7) months, and the 2-year and 5-year survival rates were 31.1\% (LD 48.1\%, ED 17.7\%) and 9.5\% (LD |1.1\%, ED 5.9\%), respectively. Grade 3 or 4 leucopenia, neutropenia, thrombocytopenia, anaemia, and diarrhoea occurred in 33, 74, 4I, 39, and I3\% of cases, respectively. In conclusion, the combination of irinotecan and carboplatin is an active and well-tolerated regimen in cases of SCLC.

British Journal of Cancer (2006) 94, 1267-127I. doi:I0.1038/sj.bjc.6603079 www.bjcancer.com

Published online 4 April 2006

(c) 2006 Cancer Research UK

Keywords: small-cell lung cancer; chemotherapy; clinical trial; irinotecan; carboplatin

Irinotecan hydrochloride is a water-soluble prodrug that is metabolized to the active metabolite SN-38, which inhibits the function of DNA topoisomerase I in cancer cells (Hsiang and Liu, 1988; Hertzberg et al, 1989). Irinotecan displays antitumour activity in various cancer cells in vitro, and has been used in the treatment of human cancers including lung cancer (Masuda et al, 1996). Preclinical studies demonstrated synergism and noncross resistance between platinum agents and irinotecan or SN-38 (Kanzawa et al, 1990; Kano et al, 1993; Fukuda et al, 1996). In small-cell lung cancer (SCLC), clinical trials with cisplatin and weekly doses of irinotecan yielded a relatively high response rate of $84 \%$ (Kudoh et al, 1998) and demonstrated effectiveness in a recent phase III study (Noda et al, 2002).

Carboplatin is a platinum derivative with less renal toxicity, and to which caused less nausea and vomiting than cisplatin (Rose and Schurig, 1985; Lokich and Anderson, 1998), and has been combined with other newer agents in chemotherapy for SCLC (Go and Adjei, 1999; Simon and Wagner, 2003). Accordingly, we conducted a phase I trial with carboplatin and weekly doses of irinotecan, and found that 11 of 13 SCLC patients had an objective response, the response rate being 85\% (complete response (CR), 31\%; partial response (PR), 54\%) (Fukuda et al, 1999). Pharma-

*Correspondence: Dr M Fukuda; E-mail: mifukuda258@nifty.com Received 25 July 2005; revised 9 March 2006; accepted 9 March 2006; published online 4 April 2006 codynamic studies have been performed to predict the clearance and administer the appropriate dose of carboplatin in individual patients (Egorin et al, 1985; Calvert et al, 1989; Chatelut et al, 1995; Duffull and Robinson, 1997; Mountain and Dresler, 1997). In a prior phase I study, we prospectively evaluated the Chatelut formula, and reported that the predicted carboplatin clearance (CL) was closely correlated with the actual CL, and the actual AUCs of carboplatin nearly reached the target AUC of 5 (Fukuda et al, 1999).

Based on these results, we conducted a phase II study of irinotecan and carboplatin therapy for SCLC. The main objectives of the study were to determine the efficacy and safety of irinotecan and carboplatin therapy in previously untreated patients with SCLC.

\section{PATIENTS AND METHODS}

The study protocol was approved by the Ethical Committee of Nagasaki University School of Medicine. This study is an independent collaborative (nonsponsored) group study.

\section{Patients}

Eligibility criteria for patients in this study included the following: a histologically confirmed diagnosis of SCLC; no prior 
chemotherapy or radiotherapy; age $\leqslant 75$ years; Eastern Cooperative Oncology Group (ECOG) performance status (PS) $\leqslant 2$; life expectancy greater than 12 weeks; adequate bone marrow function (leucocyte count $\geqslant 4000 \mu \mathrm{l}^{-1}$, platelet count $\geqslant 10.0 \times 10^{4} \mu \mathrm{l}^{-1}$, and hemoglobin level $\geqslant 10.0 \mathrm{~g} \mathrm{dl}^{-1}$ ); serum bilirubin level $\leqslant 1.25 \mathrm{mg} \mathrm{dl}^{-1}$; ALT and AST levels $\leqslant 2$ times the normal upper limit; serum creatinine level $\leqslant 10^{5} \mathrm{mg} \mathrm{dl}^{-1}$ and $\mathrm{PaO}_{2} \geqslant 70 \mathrm{mmHg}$; no medical problems severe enough to prevent compliance with the protocol; and written informed consent.

Limited disease (LD) was defined as disease confined to one hemithorax with regional lymph node metastasis, including hilar mediastinal and supraclavicular nodes, whether ipsilateral or contralateral. Extensive disease (ED) included all patients with disease sites that were not eligible for the limited-stage classification.

\section{Treatment}

Based on our phase I study (Fukuda et al, 1999), patients received $50 \mathrm{mg} \mathrm{m}^{-2}$ of irinotecan on days 1,8 , and 15 , and carboplatin with a target AUC of $5 \mathrm{mg} \mathrm{min}^{-1} \mathrm{ml}^{-1}$ on day 1 . The dose of carboplatin was determined by multiplying the target AUC of 5 by the carboplatin CL, which was predicted with the Chatelut formula (Chatelut et al, 1995) using the Jaffé method to measure serum creatinine. The value of serum creatinine obtained using the enzymatic method $(X)$ were converted to that using the Jaffé method $(Y)$ with the formula $Y=0.97 X+0.27$. Then, the estimated $\mathrm{CL}\left(\mathrm{ml} \mathrm{min}^{-1}\right)$ were calculated in those institutions as follows: $\mathrm{CL}=0.134 \times$ weight $+(218 \times$ weight $\times(1-0.00457 \times$ age $) \times(1-0.314 \times$ sex) $\times 113 \times 1.03 \times 10^{-4} /(\mathrm{s}-\mathrm{Cr}+0.276)$ (with weight in $\mathrm{kg}$, age in years, and $s e x=0$ if male and $s e x=1$ if female). In Japanese institutions, the measurement method of serum creatinine was changed to the enzymatic method. Carboplatin was administered during a $60-\mathrm{min}$ intravenous infusion of $250 \mathrm{ml}$ of $5 \%$ dextrose followed by $500 \mathrm{ml}$ of normal saline as a 2 -h infusion. This was followed by a 90 -min intravenous infusion of irinotecan in $250 \mathrm{ml}$ of $5 \%$ dextrose. Irinotecan was not administered on day 8 or 15 in the cycle if the leucocyte count was $<3 \times 10^{9} \mathrm{l}^{-1}$, the platelet count was $<100 \times 10^{9} 1^{-1}$, or the patient had diarrhoea on those days. The next cycle commenced after the leucocyte and platelet counts reached at least $3 \times 10^{9}$ and $100 \times 10^{9} 1^{-1}$, respectively. This chemotherapy was repeated every 4 weeks. The patients were planned to receive four cycles of chemotherapy. After four cycles of chemotherapy, standard thoracic irradiation at $60 \mathrm{~Gy}$ was administered to patients with $\mathrm{LD}$.

\section{Patient evaluation}

Tumour staging was performed, as described previously (Fukuda et al, 1999), using a tumour-node-metastasis system (Mountain and Dresler, 1997). Before the first cycle, a blood cell count, urinalysis, and biochemistry tests were performed to assess renal and hepatic function, and electrolytes. This monitoring was repeated during treatment, while other investigations were repeated, as necessary, to evaluate marker lesions. After the completion of treatment, each disease was assessed and tumours were restaged. The eligibility, assessability, and response of each patient were determined by extramural reviewers.

Tumour response and toxicities were classified according to World Health Organization criteria (WHO, 1979). A CR represented the disappearance of any evidence of tumours for at least 4 weeks. A PR was defined as a $50 \%$ or more reduction in the sum of the product of the greatest perpendicular diameter of all lesions for at least 4 weeks. Stable disease (SD) was defined as a $<50 \%$ reduction or $\leqslant 25 \%$ increase in the products of the greatest perpendicular diameters of all the lesions, but without any evidence of new lesions. Progressive disease (PD) was defined as an increase of $>25 \%$ or the appearance of new lesions.

\section{Statistical analyses}

The primary end point of this study was to estimate the objective response rate. The two-stage accrual design described by Simon (1987) was used. Assuming an overall response rate of $70 \%$ for standard therapy, a target response rate of $85 \%$ was established. $\alpha=0.05, \beta=0.20$, and the estimated required number of patients was more than 49 . Considering unfitness, drop out and discontinuation, the sample size of this study was determined to be 60 . Overall survival was calculated by the Kaplan--Meier method (Kaplan and Meier, 1958).

\section{RESULTS}

A total of 61 patients from 10 institutions were enrolled in this trial, between January 1998 and November 2000. All patients received irinotecan/carboplatin therapy and were evaluated for toxicity, response, and survival. The patient characteristics are shown in Table 1. In all, 27 patients (44\%) had limited disease (LD) and 34 patients $(56 \%)$ had ED.

\section{Treatment administration}

A total of 194 cycles of this therapy were administered: one cycle in six patients, two cycles in 11 , three in 13 , four in 28 , and five in three patients. In all, 37 irinotecan administrations were skipped on day 8,55 on day 15 , and 16 on both days. The major reasons for omissions on days 8 and 15 were leucopenia 47 cases, diarrhoea 16 cases, thrombocytopenia nine cases, leucopenia/diarrhoea and leucopenia/thrombocytopenia four cases and reduction in PS three cases. The administration rate (actual/planned number of treatment) of irinotecan on days 8 and 15 was 81 and $72 \%$, respectively. The average treatment delay was 1.4 days per cycle. The dose intensity (DI) of irinotecan was $30.0 \mathrm{mg} \mathrm{m}^{-2}$ week $^{-1}$, which was $80.0 \%$ of the projected DI. The DI rate relative to the projected DI of carboplatin was $95.4 \%$.

\section{Efficacy}

All 61 patients were evaluated for response. Objective tumour response was observed in 51 patients with 17 complete and $34 \mathrm{PR}$, with an overall response rate of $84 \%$ (95\% confidence interval (CI): $72-91 \%$ ) and CR rate of $28 \%$ (95\% CI: $17-41 \%$ ) (Table 2). Stable disease was observed in nine $(15 \%)$ patients, and the remaining one $(2 \%)$ patient had PD.

The overall survival of all 61 patients is shown in Figure 1. The median potential follow-up time was 55.0 (range, 40.1-73.5) months. The median follow-up for the five patients who survived was 58.8 (range, 54.8-63.5) months. The other 56 patients died

Table I Patient characteristics $(n=6 \mathrm{I})$

\begin{tabular}{lccc}
\hline Characteristics & LD & ED & Total \\
\hline No. of patients & 27 & 34 & 61 \\
Age (year) & & & \\
$\quad$ Median & 67 & 69 & 68 \\
Range & $51-74$ & $52-75$ & $51-75$ \\
Sex & & & \\
Male & 24 & 32 & 56 \\
Female & 3 & 2 & 5 \\
PS (ECOG) & & & \\
0 & 12 & 7 & 19 \\
I & 14 & 24 & 38 \\
2 & 1 & 3 & 4 \\
\hline
\end{tabular}

PS = performance status; ECOG = Eastern Cooperative Oncology Group. 
Table 2 Response

\begin{tabular}{lccccccc}
\hline Stage & $\boldsymbol{n}$ & CR & PR & SD & PD & RR (\%) & CR rate (\%) \\
\hline LD & 27 & 10 & 14 & 2 & $\mid$ & 89 & 37 \\
ED & 34 & 7 & 20 & 7 & 0 & 79 & 21 \\
Total & 61 & 17 & 34 & 9 & 1 & 84 & 28 \\
\hline
\end{tabular}

$\mathrm{CR}=$ complete response; $\mathrm{PR}=$ partial response; $\mathrm{SD}=$ stable disease; $\mathrm{PD}=$ progressive disease; $R R=$ response rate; $L D=$ limited disease; $E D=$ extensive disease

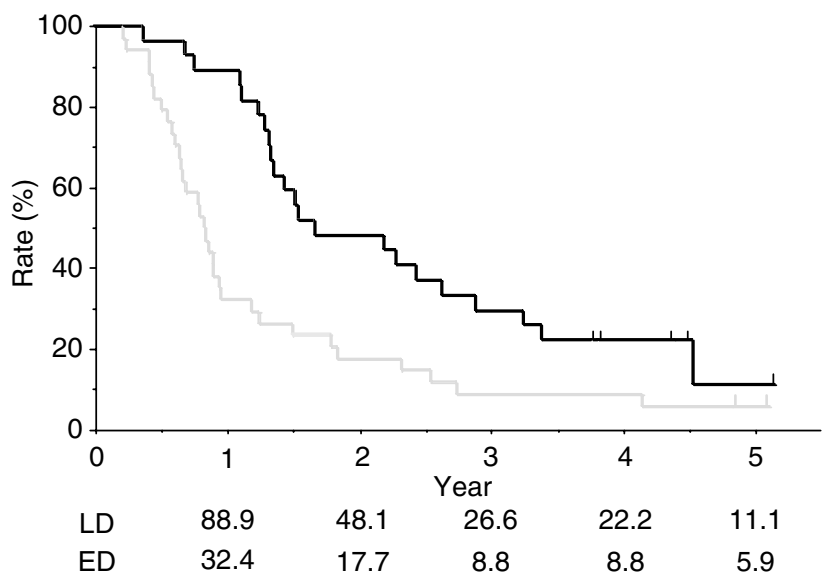

Figure I Overall survival curve of the 61 patients enrolled in the present study. A solid (_ line show the curve of 27 patients with LD, and dotted ( $\ldots$ - $)$ lines show the curve of 34 patients with ED.

Table 3 Toxicities $(n=61)$

\begin{tabular}{|c|c|c|c|c|c|}
\hline \multirow[b]{2}{*}{ Adverse event } & \multicolumn{4}{|c|}{ WHO grade $(n)$} & \multirow[b]{2}{*}{ Grade $3 / 4(\%)$} \\
\hline & $\mathbf{I}$ & 2 & 3 & 4 & \\
\hline \multicolumn{6}{|l|}{ Haematological } \\
\hline Anaemia & 13 & 18 & 18 & 6 & 39 \\
\hline Leucopenia & 7 & 30 & 16 & 4 & 33 \\
\hline Neutropenia & 2 & 11 & 28 & 17 & 74 \\
\hline Thrombocytopenia & 15 & 11 & 16 & 9 & 41 \\
\hline \multicolumn{6}{|l|}{ Nonhaematological } \\
\hline Diarrhoea & 21 & 12 & 5 & 3 & 13 \\
\hline Elevated transaminases & 8 & 2 & 0 & 0 & 0 \\
\hline Nausea/vomiting & 27 & 13 & 5 & - & 8 \\
\hline Fever & 10 & 7 & 0 & 0 & 0 \\
\hline Alopecia & 33 & 13 & 0 & 0 & 0 \\
\hline Pneumonitis & । & 0 & । & 0 & 2 \\
\hline
\end{tabular}

during the follow-up period. Median time to tumour progression was 6.1 (LD 6.4, ED 5.4) months. Median survival time (MST) was 15.0 (LD 20.0, ED 9.7) months, and the 1-, 2-, 3-, 4-, and 5-year survival rates were $57.4,31.1,18.0,14.8$, and $9.5 \%$, respectively. Survival rates for each stage are also shown in Figure 1.

\section{Toxicity}

The toxicities noted during the treatment are listed in Table 3. All 61 patients were assessable for toxicity. In all, $51(84 \%)$ patients experienced grade 3 or 4 haematological toxicity, and 21 (34\%) had grade 4 . The principal grade 3 or 4 haematological toxicity was neutropenia in $45(74 \%)$ patients, and the principal grade 4 toxicities were neutropenia and thrombocytopenia in $17(28 \%)$ and nine $(15 \%)$ patients, respectively. Febrile neutropenia occurred in five (8\%) patients. Concerning nonhaematological toxicity, eight (13\%) patients experienced grade 3 or 4 diarrhoea, one of whom had grade 4 with fever and grade 3 ileus during the first cycle and was obliged to finish with the treatment. Interstitial pneumonitis occurred in one (2\%) patient, who had a high fever for 2 days from day 24 of the third cycle. This patient had hypoxaemia with ground-glass opacity in their chest X-ray on day 28. The patient improved with corticosteroid therapy. No other severe toxicities were observed in the liver, kidney, nervous system, urinary bladder, skin, or mucous membrane.

\section{DISCUSSION}

In the present trial of irinotecan/carboplatin therapy for SCLC, the overall and CR rates, MST, and 2-year survival rate were 84 , $28,15.0$ months, and $31.1 \%$, respectively. Although these results indicate that irinotecan/carboplatin have comparable activity with irinotecan/cisplatin, which yielded values of 84, 29, 13.2 months, and $19.3 \%$, respectively, for SCLC (Kudoh et al, 1998), the results differed with each stage.

In previously untreated ED-SCLC patients, platinum-based chemotherapy remains the mainstay. A recent Japan Clinical Oncology Group (JCOG) phase III study (Noda et al, 2002) revealed that patients randomized to the irinotecan/cisplatin arm did significantly better than the group randomized to the cisplatin/ etoposide arm (MST 12.8 vs 9.4 months; 2-year survival rate 19.5 vs $5.2 \%$, respectively), and revived the good survival results of the irinotecan/cisplatin phase II study (MST 13.0 months and 2-year survival rate $21.7 \%$ in ED) (Kudoh et al, 1998). The MST of the present study in ED patients, 9.7 months, fell short of that of irinotecan/cisplatin, and was near that of cisplatin/etoposide. It is encouraging that the patients were older (median age, 69 vs 63 years) and included fewer females (6 vs 14-26\%) than in these other studies, and the 2-year survival rate $(17.7 \%)$ and response rate $(79 \%)$ were good and similar to those for irinotecan/cisplatin rather than cisplatin/etoposide. Recent large randomized trials of cisplatin/etoposide with or without newer cytotoxic agents have demonstrated a MST of 8.0-10.6 months in previously untreated patients with ED-SCLC (De Marinis et al, 2003, Georgoulias et al, 2004; Niell et al, 2005). In addition, a recent meta-analysis of randomized trials demonstrated that patients who received a regimen containing cisplatin had a significant increase in the probability of response and survival (Pujol et al, 2000; Hotta et al, 2004). Thus, we consider irinotecan/carboplatin of value as a convenient carboplatin-containing two-drugs regimen with similar activity to cisplatin-containing regimens, except irinotecan/cisplatin in patients with ED-SCLC. More recently, a multicentre, large randomized phase III trial $(n=331)$ was conducted to confirm the results of the JCOG study (Hanna et al, 2005). The patients were randomized to either irinotecan/cisplatin or cisplatin/etoposide and there was no statistically significant difference in overall survival (MST 9.3 vs 10.2 months). The DI delivered of irinotecan was higher in this trial ( $39 v s 36.2 \mathrm{mg} \mathrm{m}^{-2} \mathrm{wk}^{-1}$ ), however, the DI $\left(\mathrm{mg} \mathrm{m}^{-2} \mathrm{wk}^{-1}\right)$ rate of irinotecan to cisplatin were lower $(39 / 18=2.17$ vs $36.2 / 14.3=2.53)$, and the response rate of $48 \%$ in irinotecan/cisplatin arm of this study was lower than that of the JCOG $(84.4 \%)$ and our study (79\%). Thus, we consider that the insufficiency use of irinotecan in Hanna's-modified irinotecan/ cisplatin regimen or the ethnic difference might have caused the study failure.

In previously untreated LD-SCLC, it has emerged that concurrent thoracic chemoradiotherapy (CRT) is superior to sequential CRT when platinum/etoposide regimens are used for chemotherapy (Simon and Wagner, 2003). When the irinotecan/ cisplatin regimen was used, ordinary concurrent CRT was deemed unacceptable (Yokoyama et al, 1998), and unexpectedly poor results of the sequential CRT in LD-SCLC with a MST of 14.3 
months and 2-year survival rate of $17.5 \%$ (Kudoh et al, 1998) may even suggest unsuitableness of irinotecan/platinum for radiotherapy. To resolve this issue, we administered a split-course early concurrent radiotherapy combined with irinotecan/cisplatin (Oka et al, 2001, 2002; Nagashima et al, 2004). Although the phase II study in LD-SCLC is not finished yet, the MST and 2-year survival rate were 25.2 months and $56.2 \%$, respectively, in our phase I study (Oka et al, 2002). Alternatively, we have used late sequential radiotherapy combined with irinotecan/carboplatin. The MST in the present study did not reach that obtained with cisplatin/ etoposide or in early concurrent radiotherapy studies (Takada et al, 2002; Segawa et al, 2003) but was similar to values in other cooperative studies (Hanna et al, 2002; Edelman et al, 2004; Schild et al, 2004) and the 2-year survival rate nearly reached the level obtained in two early concurrent studies (Takada et al, 2002; Segawa et al, 2003). Furthermore, the present study included rather advanced cases of LD in which more than half of the lung fields were affected, and in some ways, these cases are a good indication of the present regimen in practical use.

\section{REFERENCES}

Calvert AH, Newell DR, Gumbrell LA, O'Reilly S, Burnell M, Boxall FE, Siddik ZH, Judson IR, Gore ME, Wiltshaw E (1989) Carboplatin dosage: prospective evaluation of a simple formula based on renal function. J Clin Oncol 7: 1748-1756

Chatelut E, Canal P, Brunner V, Chevreau C, Pujol A, Boneu A, Roche $\mathrm{H}$ Houin G, Bogat R (1995) Prediction of carboplatin clearance from standard morphological and biological patient characteristics. $J$ Natl Cancer Inst 87: 573-580

De Marinis F, Migliorino MR, Paoluzzi L, Portalone L, Ariganello O, Cortesi E, Gamucci T, Gasperoni S, Cipri A, Martelli O, Nelli F (2003) Phase I/II trial of gemcitabine plus cisplatin and etoposide in patients with smallcell lung cancer. Lung Cancer 39: 331-338

Duffull SB, Robinson BA (1997) Clinical pharmacokinetics and dose optimisation of carboplatin. Clin Pharmacokinet 33: 161-183

Edelman MJ, Chansky K, Gaspar LE, Leigh B, Weiss GR, Taylor SA, Crowley J, Livingston R, Gandara DR (2004) Phase II trial of cisplatin/etoposide and concurrent radiotherapy followed by paclitaxel/carboplatin consolidation for limited small-cell lung cancer: Southwest Oncology Group 9713. J Clin Oncol 22: 127-132

Egorin MJ, Van Echo DA, Olman EA, Whitacre MY, Forrest A, Aisner J (1985) Prospective validation of a pharmacologically based dosing schema for the cis-diamminedichloroplatinum (II) analogue diamminecyclobutanedicarboxylato-platinum. Cancer Res 45: 6502-6506

Fukuda M, Nishio K, Kanzawa F, Ogasawara H, Ishida T, Arioka H, Bojanowski K, Oka M, Saijo N (1996) Synergism between cisplatin and topoisomerase I inhibitors, NB-506 and SN-38, in a human small cell lung cancer cells. Cancer Res 56: 789-793

Fukuda M, Oka M, Soda H, Terashi K, Kawabata S, Nakatomi K, Takatani H, Tsurutani J, Tsukamoto K, Noguchi Y, Fukuda M, Kinoshita A, Kohno S (1999) Phase I study of irinotecan combined with carboplatin in previously untreated solid cancers. Clin Cancer Res 5: 3936-3969

Fukuda M, Oka M, Soda H, Kinoshita A, Fukuda M, Nagashima S, Kuba M, Takatani H, Tsurutani J, Nakamura Y, Kasai T, Inoue Y, Soejima Y, Kohno S (2004) Phase II study of irinotecan combined with carboplatin in previously untreated non-small cell lung cancer. Cancer Chemother Pharmacol 54: $573-577$

Georgoulias V, Mavroudis D, Veslemes M, Papakotoulas P, Syrigos K, Agelidou A, Pavlakou G, Rapti A, Polyzos A (2004) Comparison of the sequential versus the alternate administration of cisplatin-etoposide and topotecan regimens as first line treatment in extensive stage small cell lung cancer (SCLC): a preliminary analysis of a multicenter randomized trial. Proc Am Soc Clin Oncol 23: 624 (abstr 7045)

Go RS, Adjei AA (1999) Review of the comparative pharmacology and clinical activity of cisplatin and carboplatin. J Clin Oncol 17: 409-422

Hanna N, Ansari R, Fisher W, Shen J, Jung SH, Sandler A (2002) Etoposide, ifosfamide and cisplatin (VIP) plus concurrent radiotherapy for previously untreated limited small cell lung cancer (SCLC): a Hoosier Oncology Group (HOG) phase II study. Lung Cancer 35: 293-297
The main toxicity of our irinotecan/carboplatin therapy was haematological. Comparing grade 3 or higher toxicities in the present study with those for non-small-cell lung cancer (Fukuda et $a l, 2004)$, the incidences of neutropenia, thrombocytopenia, anaemia, and diarrhoea were higher $(74,41,39,13$ vs $60,25,32$, and $7 \%$, respectively), and those of leucopenia, nausea/vomiting, and pneumonitis were comparable $(33,8,2$ vs $32,10,2 \%$, respectively). This difference was caused by the difference in therapeutic period between SCLC and NSCLC. Platelet transfusion was performed only twice in 136 cycles of irinotecan/carboplatin therapy. Comparing grade 3 or higher toxicities with irinotecan/ cisplatin (Noda et al, 2002; Takada et al, 2002), the incidence of thrombocytopenia was higher, and the incidences of nausea/ vomiting and diarrhoea were lower in the present study.

In conclusion, our multicentre phase II trial demonstrated the usefulness of irinotecan/carboplatin in chemotherapy for SCLC. This regimen is a convenient noncisplatin treatment comparable in terns of the probability of response and survival with other regimens for SCLC.
Hanna NH, Einhorn L, Sandler A, Langer C, Hariharan S, Ansari R, Ellis P, Byrne M, Wang B, Bunn P (2005) Randomized, phase III trial comparing irinotecan/cisplatin (IP) with etoposide/cisplatin (EP) in patients (pts) with previously untreated, extensive-stage (ES) small cell lung cancer (SCLC). Proc Am Soc Clin Oncol 23: 622s (abstr 7004)

Hertzberg RP, Caranfa MJ, Holden KG, Jakas DR, Gallagher G, Mattern MR, Mong SM, Bartus JO, Johnson RK, Kingsbury WD (1989) Modification of the hydroxyl lactone ring of camptothecin: inhibition of mammalian topoisomerase I and biological activity. J Med Chem 32: 715-720

Hotta K, Matsuo K, Ueoka H, Kiura K, Tabata M, Tanimoto M (2004) Meta-analysis of randomized clinical trials comparing cisplatin to carboplatin in patients with advanced non-small-cell lung cancer. J Clin Oncol 22: 3852-3859

Hsiang YH, Liu LF (1988) Identification of mammalian DNA topoisomerase I as an intracellular target of the anticancer drug camptothecin. Cancer Res 48: $1722-1726$

Kano Y, Akutsu M, Suzuki K, Yoshida M (1993) Effects of carboplatin in combination with other anticancer agents on human leukemia cell lines. Leuk Res 17: 113-119

Kanzawa F, Sugimoto Y, Minato K, Kasahara K, Bungo M, Nakagawa K, Fujiwara Y, Liu LF, Saijo N (1990) Establishment of a camptothecin analogue (CPT-11)-resistant cell line of human non-small cell lung cancer: characterization and mechanism of resistance. Cancer Res 50: 5919-5924

Kaplan E, Meier P (1958) Nonparametric estimation from incomplete observations. J Am Stat Assoc 53: 457-481

Kudoh S, Fujiwara Y, Takada Y, Yamamoto H, Kinoshita A, Ariyoshi Y, Furuse K, Fukuoka M (1998) Phase II study of irinotecan combined with cisplatin in patients with previously untreated small-cell lung cancer. J Clin Oncol 16: $1068-1074$

Lokich J, Anderson N (1998) Carboplatin versus cisplatin in solid tumors: an analysis of the literature. Ann Oncol 9: 13-21

Masuda N, Kudoh S, Fukuoka M (1996) Irinotecan (CPT-11): pharmacology and clinical applications. Crit Rev Oncol/Hematol 24: 3-26

Mountain CF, Dresler CM (1997) Regional lymph node classification for lung cancer staging. Chest 111: $1718-1723$

Nagashima S, Fukuda M, Kinoshita A, Fukuda M, Kasai T, Takatani $\mathrm{H}$, Rikimaru T, Soda H, Oka M, Kohno S (2004) Phase II study of irinotecan (CPT-11) and cisplatin (CDDP) with concurrent split-course thoracic radiotherapy (TRT) in stage III non-small cell lung cancer (NSCLC). Proc Am Soc Clin Oncol 23: 655 (abstr 7169)

Niell HB, Herndon JE, Miller AA, Watson DM, Sandler AB, Kelly K, Marks RS, Perry MC, Ansari RH, Otterson G, Ellerton J, Vokes EE, Green MR (2005) Randomized phase III intergroup trial of etoposide and cisplatin with or without paclitaxel and granulocyte colony stimulating factor in patients with extensive-stage small-cell lung cancer: Cancer and Leukemia Group B Trial 9732. J Clin Oncol 23: 3752-3759

Noda K, Nishiwaki Y, Kawahara M, Negoro S, Sugiura T, Yokoyama A, Fukuoka M, Mori K, Watanabe K, Tamura T, Yamamoto S, Saijo N 
(2002) Irinotecan plus cisplatin compared with etoposide plus cisplatin for extensive small-cell lung cancer. $N$ Engl J Med 346: 85-91

Oka M, Fukuda M, Fukuda M, Kinoshita A, Kuba M, Ichiki M, Rikimaru T, Soda H, Takatani H, Narasaki F, Nagashima S, Nakamura Y, Hayashi N, Kohno S (2001) Phase I study of irinotecan and cisplatin with concurrent split-course radiotherapy in unresectable and locally advanced non-small cell lung cancer. Eur J Cancer 37: 1359-1365

Oka M, Fukuda M, Kuba M, Ichiki M, Rikimaru T, Soda H, Tsurutani J, Nakamura Y, Kawabata S, Nakatomi K, Narasaki F, Nagashima S, Takatani H, Fukuda M, Kinoshita A, Kohno S (2002) Phase I study of irinotecan and cisplatin with concurrent split-course radiotherapy in limited-disease small-cell lung cancer. Eur I Cancer 38: 1998-2004

Pujol JL, Carestia L, Daures JP (2000) Is there a case for cisplatin in the treatment of small-cell lung cancer? a meta-analysis of randomized trials of a cisplatin-containing regimen $v s$ a regimen without this alkylating agent. Br J Cancer 83: 8-15

Rose WC, Schurig JE (1985) Preclinical antitumor and toxicologic profile of carboplatin. Cancer Treat Rev 12: 1-19

Schild SE, Bonner JA, Shanahan TG, Brooks BJ, Marks RS, Geyer SM, Hillman SL, Farr Jr GH, Tazelaar HD, Krook JE, Geoffroy FJ, Salim M, Arusell RM, Mailliard JA, Schaefer PL, Jett JR (2004) Long-term results of a phase III trial comparing once-daily radiotherapy with twice-daily

\section{Appendix}

\section{Principal NTOG Investigators in this Trial}

Tetsuya Iida, Mikio Oka, Takashi Kasai, Shigeru Kawabata, Tetsuro Kanda, Shigeru Kohno, Takeshi Kitazaki, Akitoshi radiotherapy in limited-stage small-cell lung cancer. Int J Radiat Oncol Biol Phys 59: $943-951$

Segawa Y, Ueoka H, Kiura K, Tabata M, Takigawa N, Hiraki Y, Watanabe Y, Yonei T, Moritaka T, Hiyama J, Hiraki S, Tanimoto M, Harada M (2003) Phase I/II study of altered schedule of cisplatin and etoposide administration and concurrent accelerated hyperfractionated thoracic radiotherapy for limited-stage small-cell lung cancer. Lung Cancer 41: 13-20 Simon GR, Wagner H (2003) Small cell lung cancer. Chest 123: 259S - 271S Simon R (1987) How large should be a phase II trial of a new drug? Cancer Treat Rep 71: 1079-1085

Takada M, Fukuoka M, Kawahara M, Sugiura T, Yokoyama A, Yokota S, Nishiwaki Y, Watanabe K, Noda K, Tamura T, Fukuda H, Saijo N (2002) Phase III study of concurrent versus sequential thoracic radiotherapy in combination with cisplatin and etoposide for limited-stage small-cell lung cancer: results of the Japan Clinical Oncology Group Study 9104. $J$ Clin Oncol 20: $3054-3060$

WHO (1979) WHO Handbook for Reporting Results of Cancer Treatment. Geneva: WHO

Yokoyama A, Kurita Y, Saijo N, Tamura T, Noda K, Shimokata K, Matsuda $\mathrm{T}$ (1998) Dose-finding study of irinotecan and cisplatin plus concurrent radiotherapy for unresectable stage III non-small-cell lung cancer. $\mathrm{Br} \mathrm{J}$ Cancer 78: $257-262$

Kinoshita, Mutsuo Kuba, Yoshifumi Soejima, Hiroshi Soda, Hiroshi Takatani, Nanae Tomonaga, Junji Tsurutani, Seiji Doi, Seiji Nagashima, Katsumi Nakatomi, Hirofumi Nakano, Reiji Nakano, Yoichi Nakamura, Masaaki Fukuda, Minoru Fukuda, Satoru Fujino, and Hiroyuki Yamaguchi. 